\title{
Physico-Chemical Assessment of Drinking Water in Urban and Peri-Urban Areas of Udaipur, India
}

\author{
Nirmal Kumar ${ }^{1 *}$, Abhishek Gaurav ${ }^{1}$, Surendra Singh Shekhawat ${ }^{1}$, \\ Bincy Joseph ${ }^{2}$, Hitesh Kumar ${ }^{1}$ and Devender Choudhary ${ }^{1}$
}

Veterinary Public Health and Epidemiology Department, College of Veterinary And Animal Sciences, Navania, Vallabhnagar, Udaipur, Rajasthan, India

*Corresponding author:

\section{A B S T R A C T}

\section{Keywords \\ Drinking water, Physico-chemical, Urban and peri- urban areas, Bureau of Indian Standards \\ Article Info \\ Accepted: \\ 20 July 2019 \\ Available Online: \\ 10 August 2019}

\begin{abstract}
The quality of drinking water is an important environmental determinant of health. In present study, carried out of physico-chemical analysis of drinking water like public drinking water, surface water, ground water and animal drinking water were collected from different parts of urban and peri-urban areas of Udaipur. Parameters such as $\mathrm{pH}$, electrical conductivity, turbidity, total dissolved solids (TDS), total hardness, nitrate, fluoride, iron, chloride and residual free chlorine were analyzed and range were founded 6.80 to $8.62,82 \mu \mathrm{s} / \mathrm{cm}$ to $5430 \mu \mathrm{s} / \mathrm{cm}, 0 \mathrm{NTU}$ to $25 \mathrm{NTU}, 41 \mathrm{mg} / \mathrm{l}$ to $2715 \mathrm{mg} / \mathrm{l}, 25 \mathrm{mg} / \mathrm{l}$ to $1925 \mathrm{mg} / \mathrm{l}, 0 \mathrm{mg} / \mathrm{l}$ to $100 \mathrm{mg} / \mathrm{l}, 0 \mathrm{mg} / \mathrm{l}$ to $2.5 \mathrm{mg} / \mathrm{l}, 0 \mathrm{mg} / \mathrm{l}$ to $1.0 \mathrm{mg} / \mathrm{l}, 10 \mathrm{mg} / \mathrm{l}$ to $1100 \mathrm{mg} / \mathrm{l}$ and $0 \mathrm{mg} / \mathrm{l}$, respectively. Results showed that the most of the parameters were exceeded the recommended drinking water quality levels of Bureau of Indian Standards (BIS, 2012). Results indicated most of drinking water is not to be suitable for consumptions and recommended to treated before consumptions.
\end{abstract}

\section{Introduction}

Clean and safe drinking water are essential for health, survival, growth and development. But, in developing country like India, still there are some regions where the basic necessities of drinking water are not available. Provision of clean and safe water to the population will not only reduce the expenditure incurred on the health services but will also spur economic growth. Improved water supply and sanitation, and better management of water resources, can boost countries' economic growth and can contribute greatly to poverty reduction (WHO, 2017). Water within the distribution system (such as leaky pipe or outdated infrastructure) or of stored domestic water as a result of unhygienic handling (WHO, 2010).

These physico-chemical parameters indicates the deterioration of water quality which is the result of various anthropogenic disturbances like industrialization, construction activities, utilization of agricultural and forest land for other developmental purposes. The pollution of these water bodies primarily affects the chemical quality and then systematically 
destroys the community disturbing the delicate balance of food chain. Physicochemical qualities of these water bodies as source of drinking water is necessitated by the presence of dead vegetation, heavy metal leachates from solid waste dump, domestic and industrial sewages, surface runoffs from agricultural farms etc. (Ademola, 2008).

The inappropriate supervision of water systems leads to severe problems in accessibility and quality of water. The world is growing at a very fast pace with its technologies and the population on earth is increasing tremendously. So, the dependence as well as exploitation of water resources is also increasing rapidly. It is not just the population increase alone but also the technology-aided excessive uses, abuses and misuses of water resources that break the natural water cycle. The water quality description is denoted by assessing the physical parameters like $\mathrm{pH}$, TDS (total dissolved solids), TSS (total suspended solids) and chemical parameters like total alkalinity, free $\mathrm{CO}_{2}$, DO (dissolved oxygen), total hardness, $\mathrm{Ca}, \mathrm{Mg}$, salinity and bacterial parameters like SPC (standard plate count), TCC (total coliform count), etc.

Groundwater forms a vital supply of drinking water supply for urban and rural people of India. There are several states in India where more than $90 \%$ population are dependent directly on ground water for drinking and other purposes (Ramachandraiah, 2004). In India, almost $70 \%$ of water has become polluted due to the discharge of household sewage and industrial effluents into the natural water sources, like rivers, streams and lakes (Sangu and Sharma 1987).

\section{Materials and Methods}

In the present study, attempts were made to assess the physico-chemical quality of drinking water in urban and peri-urban areas of Udaipur (Rajasthan) over a period of June 2018 to January 2019. Udaipur is located in the southern part of Rajasthan. It is actually lying in the center of a bowl-shaped basin surrounded by the Aravali hills and is drained by the Ayad river.

\section{Collection of samples}

Four different category of 85 water samples (public drinking water $\mathrm{n}=23$, surface water $n=22$, ground water $n=20$ and animal drinking water $\mathrm{n}=20$ ) were collected in $1000 \mathrm{ml}$ caped glass bottle and brought to the Laboratory of Environmental Hygiene in chilled condition, Department of Veterinary Public Health \& Epidemiology, CVAS, Navania, Vallabhnagar, Udaipur and processed within 4-6 hrs of collection.

\section{pH determination}

$\mathrm{pH}$ is determined by digital $\mathrm{pH}$ meter (Chino),

\section{Total dissolved solid determination}

TDS of water samples were analysed by digital meter (Divinext digital meter, Balram enterprises, Ludhiana).

\section{Electrical conductivity determination}

EC were assessed by digital conductivity meter 304 systronics UVSAR, India.

\section{Total Hardness of water $\left(\mathrm{as}^{\mathrm{CaCO}} 3\right)$}

Total hardness of water samples were determined by ethylene diamine tetra acetic acid (EDTA) titration method.50 $\mathrm{ml}$ of water sample was taken without dilution in the porcelain dish or conical flask and $1 \mathrm{ml}$ of buffer solution and $1 \mathrm{ml}$ of inhibitor solution. (for monitoring interference from aluminum and manganese) were added then indicator 
erichrome black-T was added and titrated it against the standard EDTA solution till the color of solution changed from red to blue, showing the end-point of titration. The volume of EDTA consumed was recorded during the titrations as $\mathrm{V}_{1}(\mathrm{ml})$.Same amount $(50 \mathrm{ml})$ of deionized distilled water was taken and $1 \mathrm{ml}$ buffer solution, $1 \mathrm{ml}$ of inhibitor solution and indicator erichrome black-T were added and titrated against the standard EDTA solution in the same manner the volume of titrated EDTA consumed was recorded as $V_{2}$ $(\mathrm{ml})$.

Net volume of EDTA solution required by water sample was $\mathbf{V}=\mathbf{V}_{\mathbf{1}}-\mathbf{V}_{\mathbf{2}}(\mathbf{m l})$

The hardness was calculated by the formula given below:

\section{Calculation}

Total Hardness $\left(\right.$ as $\left.\mathrm{CaCO}_{3} \mathrm{mg} / \mathrm{l}\right)=$

$\mathrm{V}(\mathrm{ml}) \times 1000$

Volume of water sample before dilution(in ml)

Nitrate, fluoride, iron and chloride

Estimated according to the procedure prescribed by American Public Health Association (APHA, 2005).

\section{Turbidity and residual free chlorine}

Estimated by Himedia WT023 Octo aqua test kit.

\section{Results and Discussion}

Water is considered as potable, if it meets the recommended criteria for physical, chemical and microbiological quality set by regulatory agencies. Potable water is required for good health and socio-economic development of man and animal.The acceptability and use of potable water for recreational and other domestic needs are influenced by physicochemical parameters such as $\mathrm{pH}$, total dissolved solids and conductivity etc. Inorganic minerals however constitute the greatest source of raw water contaminants, of which mineral salts are introduced as water moves over the soil structure. A major factor affecting water quality is anthropogenic activities arising from rapid industrialization and urbanization (Ubalua and Ezeronye 2005).

$\mathrm{pH}$ of water is an important environmental factor, the fluctuation of $\mathrm{pH}$ is linked with chemical changes, species composition and life processes. It is generally considered as an index for suitability of the environment (Rani et al., 2012).In current study $\mathrm{pH}$ values found in the range between 6.80 to 8.62. In which urban areas ranged found in between 6.80 to 8.62 while, in peri-urban areas ranged found in between 6.80 to 8.62.Most of the samples comes in the acceptable range given by (BIS, 2012). Similar to our result finding of $\mathrm{pH}$ range were also reported by Pathak et al., 2016, Rai and Chouhan 2017and Ghosh, 2018. While higher $\mathrm{pH}$ range was reported by Dixit et al.,2015. Also, slightly lower $\mathrm{pH}$ value for water samples was reported by Samuel et al., 2017and Sunday et al., 2014.Variation in $\mathrm{pH}$ affects aquatic life and mainly occurs due to the different physicochemical nature of the soil. Acidic water also leads to corrosion of water pipes while the alkaline waters are less corrosive but may have bitter or soda like taste. High values of $\mathrm{pH}$ may occur due to the discharge of waste and microbial decomposition of organic matter present in water (Table 1 to 8 ; Fig. 1 and 2).

Total dissolved solids are a measure of total inorganic substances dissolved in water (ANZECC, 2000). TDS indicates the general nature of water quality or salinity. The TDS 
concentration was found to be above the permissible limit may be due to the leaching of various pollutants into the ground water which can decrease the portability and may cause gastrointestinal irritation in human and may also have laxative effect particularly upon transits (WHO, 1997). It affects the taste of water. The WHO has recommended the TDS value of $500 \mathrm{mg} / \mathrm{l}$ as acceptable for consumption. High levels of the TDS in drinking water may have adverse effect on human health due to the presence of excessive salts. While, extremely low TDS leads to the development of flat insipid taste in water. TDS values found in the range between 41 $\mathrm{mg} / \mathrm{l}$ to $2715 \mathrm{mg} / \mathrm{l}$ in different categories of water. In which urban areas ranged found in between $41 \mathrm{mg} / \mathrm{l}$ to $1639 \mathrm{mg} / \mathrm{l}$ while, in periurban areas ranged found between $237 \mathrm{mg} / \mathrm{l}$ to $2715 \mathrm{mg} / \mathrm{l}$ (Table 1 to 8; Fig. 1 and 2).All surface water and public drinking water comes in acceptable for consumption suggested by (BIS, 2012) but ground water and animal drinking water show higher value of TDS which is not consumable according to (BIS, 2012). Similar results were also reported by Shukla et al., 2013 and Buridi and Gedala 2014. While, lower TDS values were observed by Rahmania et al., 2015 and Sunitha et al., 2013.TDS recommendation for drinking water is $200 \mathrm{mg} / \mathrm{l}$ to $2000 \mathrm{mg} / \mathrm{l}$. (BIS, 2012).

Total hardness of water is expressed in $\mathrm{CaCO}_{3}(\mathrm{mg} / \mathrm{l})$ which includes calcium as well as magnesium hardness. Total hardness values found in the range between $25 \mathrm{mg} / \mathrm{l}$ to $1925 \mathrm{mg} / \mathrm{l}$ in different categories of water. In which urban areas ranged found in between $25 \mathrm{mg} / \mathrm{l}$ to $800 \mathrm{mg} / \mathrm{l}$ while, in peri-urban areas ranged found between $125 \mathrm{mg} / \mathrm{l}$ to $1925 \mathrm{mg} / \mathrm{l}$ (Table 1 to 8 ; Fig. 1 and 2).High levels of hardness in the study area in ground water and animal drinking water might be contributed due to the lime stone, zinc and magnesium rich soil. Increased values of hardness leads to scale formation in pipes which leads to their damage and leads to increased expenditure on their maintenance. It is also a hazard for human health, especially for persons suffering from kidney stones. In urban public drinking water show consumable hard water as per BIS, 2012 because of good water supply system and in other hand periurban public drinking water show more than $600 \mathrm{mg} / \mathrm{l}$ which exceed limit cause of poor public drinking water supply.Some researchers show contrasting findings were reported by Chindo et al., 2013, Mostafa et al., 2013, Buridi and Gedala 2014 and Ehiowemwenguan et al., 2014. Similar findings were also reported by Chidinma et al., 2016, Olatayo, 2014, Sebiawu et al., 2014, Chaubey and Patil 2015, Hassan et al., 2016 and Reda, 2016.Total hardness recommendation for drinking water is 200 $\mathrm{mg} / \mathrm{l}$ to $600 \mathrm{mg} / \mathrm{l}$. (BIS, 2012).

Electrical conductivity (EC) is the ability of an aqueous solution to conduct the electric current. Electrical Conductivity is a useful tool to evaluate the purity of water (Acharya et al., 2008).Electrical conductivity (EC) is the ability of a solution to conduct an electrical current which is dependent on the quantity and charge of the ions in the solution. ECvalues found in the range between 82 $\mu \mathrm{s} / \mathrm{cm}$ to $5430 \mu \mathrm{s} / \mathrm{cm}$ in different categories of water. In which urban areas ranged found in between $82 \mu \mathrm{s} / \mathrm{cm}$ to $3278 \mu \mathrm{s} / \mathrm{cm}$ while, in peri-urban areas ranged found between 474 $\mu \mathrm{s} / \mathrm{cm}$ to $5430 \mu \mathrm{s} / \mathrm{cm}$. As compared with our study, lower EC were observed by Chindo et al., 2013, Buridi and Gedala 2014, Vyas et al., 2015, Adegboyega et al., 2015 and Samuel et al., 2017. While higher EC values were reported by Saha et al., 2018. Higher EC values give an indication towards the higher concentration of mineral salts in the water. It is also due to increased corrosion of metals (Table 1 to 8; Fig. 1 and 2). 
Table.1 Physico-chemical analysis of public drinking water in urban areas of Udaipur

\begin{tabular}{|c|c|c|c|c|c|c|c|c|c|c|c|}
\hline $\begin{array}{l}\text { S. } \\
\text { No. }\end{array}$ & Sample No. & $\begin{array}{c}\mathrm{pH} \\
(\mathrm{mg} / \mathrm{L})\end{array}$ & $\begin{array}{c}\text { TDS } \\
(\mathrm{mg} / \mathrm{L})\end{array}$ & $\begin{array}{c}\mathrm{EC} \\
(\mu \mathrm{s} / \mathrm{cm})\end{array}$ & $\begin{array}{c}\text { Chloride } \\
\text { (mg/L) }\end{array}$ & $\begin{array}{c}\text { TH } \\
(\mathrm{mg} / \mathrm{L})\end{array}$ & $\begin{array}{c}\text { Fluoride } \\
\text { (mg/L) }\end{array}$ & $\begin{array}{c}\text { Iron } \\
(\mathrm{mg} / \mathrm{L})\end{array}$ & $\begin{array}{l}\text { Nitrate } \\
(\mathrm{mg} / \mathrm{L})\end{array}$ & $\begin{array}{c}\mathrm{RC} \\
(\mathrm{mg} / \mathrm{L})\end{array}$ & $\begin{array}{c}\text { Turbidity } \\
\text { (NTU) }\end{array}$ \\
\hline 1. & PW 1 & 7.52 & 473 & 947 & 80 & 275 & 1.5 & 0 & 45 & 0 & 0 \\
\hline 2. & PW 2 & 8.37 & 288 & 577 & 70 & 150 & 1.0 & 0 & 10 & 0 & 0 \\
\hline 3. & PW 3 & 7.50 & 614 & 1229 & 100 & 325 & 1.5 & 0 & 10 & 0 & 0 \\
\hline 4. & PW 4 & 7.20 & 98 & 197 & 20 & 30 & 0.0 & 0 & 0 & 0 & 0 \\
\hline 5. & PW 5 & 7.61 & 607 & 1215 & 110 & 300 & 1.5 & 0 & 10 & 0 & 0 \\
\hline 6. & PW 6 & 7.20 & 110 & 220 & 10 & 25 & 0 & 0 & 0 & 0 & 0 \\
\hline 7. & PW 7 & 6.82 & 282 & 565 & 60 & 125 & 0.5 & 0 & 10 & 0 & 0 \\
\hline 8. & PW 8 & 7.20 & 95 & 191 & 50 & 25 & 0.5 & 0 & 10 & 0 & 0 \\
\hline 9. & PW 9 & 6.90 & 671 & 1342 & 130 & 225 & 0 & 0 & 0 & 0 & 0 \\
\hline 10. & PW 10 & 7.20 & 563 & 1126 & 80 & 225 & 0.5 & 0 & 10 & 0 & 0 \\
\hline 11. & PW 11 & 6.80 & 41 & 82 & 20 & 25 & 0 & 0 & 0 & 0 & 0 \\
\hline 12. & PW 12 & 7.10 & 447 & 895 & 20 & 226 & 1.5 & 0.5 & 10 & 0 & 0 \\
\hline 13. & PW 13 & 7.20 & 352 & 704 & 30 & 194 & 1.5 & 0.3 & 10 & 0 & 0 \\
\hline 14. & Mean & 7.278 & 357 & 714.615 & 60 & 165.384 & 0.769 & 0.615 & 9.615 & 0 & 0 \\
\hline
\end{tabular}

$\mathrm{PW}=$ public water supply, $\mathrm{TDS}=$ total dissolved solid, $\mathrm{EC}=$ electrical conductivity, $\mathrm{TH}=$ total hardness, $\mathrm{RC}=$ residual chlorine

Table.2 Physico-chemical analysis of public drinking water in peri-urban areas of Udaipur

\begin{tabular}{|c|c|c|c|c|c|c|c|c|c|c|c|}
\hline S. No. & Sample No. & $\begin{array}{c}\text { pH } \\
(\mathrm{mg} / \mathrm{L})\end{array}$ & $\begin{array}{c}\text { TDS } \\
(\mathrm{mg} / \mathrm{L})\end{array}$ & $\begin{array}{c}\mathrm{EC} \\
(\mu \mathrm{s} / \mathrm{cm})\end{array}$ & $\begin{array}{c}\text { Chloride } \\
\text { (mg/L) }\end{array}$ & $\begin{array}{c}\text { TH } \\
(\mathrm{mg} / \mathrm{L})\end{array}$ & $\begin{array}{c}\text { Fluoride } \\
\text { (mg/L) }\end{array}$ & $\begin{array}{c}\text { Iron } \\
(\mathrm{mg} / \mathrm{L})\end{array}$ & $\begin{array}{c}\text { Nitrate } \\
(\mathrm{mg} / \mathrm{L})\end{array}$ & $\begin{array}{c}\text { RC } \\
(\mathrm{mg} / \mathrm{L})\end{array}$ & $\begin{array}{c}\text { Turbidity } \\
\text { (NTU) }\end{array}$ \\
\hline 1. & PW 14 & 7.50 & 946 & 1886 & 250 & 425 & 1.5 & 0.5 & 45 & 0 & 0 \\
\hline 2. & PW 15 & 7.68 & 1374 & 2748 & 410 & 475 & 1.5 & 1.0 & 10 & 0 & 5 \\
\hline 3. & PW 16 & 8.01 & 651 & 1303 & 60 & 150 & 1.5 & 0.3 & 10 & 0 & 0 \\
\hline 4. & PW 17 & 7.14 & 1787 & 3574 & 750 & 1000 & 1 & 0.3 & 10 & 0 & 0 \\
\hline 5. & PW 18 & 6.83 & 537 & 1074 & 60 & 216 & 1.0 & 0.3 & 10 & 0 & 0 \\
\hline 6. & PW 19 & 6.89 & 742 & 1448 & 110 & 300 & 0.5 & 0.3 & 10 & 0 & 0 \\
\hline 7. & PW 20 & 7.49 & 998 & 1997 & 230 & 700 & 2.0 & 0 & 10 & 0 & 0 \\
\hline 8. & PW21 & 6.90 & 1258 & 2516 & 450 & 500 & 2.0 & 0 & 0 & 0 & 0 \\
\hline 9. & PW22 & 7.26 & 500 & 1000 & 90 & 205 & 1.0 & 0.3 & 10 & 0 & 0 \\
\hline 10. & PW23 & 7.92 & 237 & 474 & 50 & 125 & 0.5 & 0 & 10 & 0 & 0 \\
\hline 11. & Mean & 7.362 & 903 & 1802 & 246 & 409.6 & 1.25 & 0.3 & 12.5 & 0 & 0.5 \\
\hline
\end{tabular}

$\mathrm{PW}=$ public water supply, $\mathrm{TDS}=$ total dissolved solid, $\mathrm{EC}=$ electrical conductivity, $\mathrm{TH}=$ total hardness, $\mathrm{RC}=$ residual chlorine 
Table.3 Physico-chemical analysis of surface water in urban areas of Udaipur

\begin{tabular}{|c|c|c|c|c|c|c|c|c|c|c|c|}
\hline $\begin{array}{l}\text { S. } \\
\text { No. }\end{array}$ & Sample No. & $\begin{array}{c}\text { pH } \\
(\mathrm{mg} / \mathrm{L})\end{array}$ & $\begin{array}{c}\text { TDS } \\
(\mathrm{mg} / \mathrm{L})\end{array}$ & $\mathrm{EC}(\boldsymbol{\mu s} / \mathbf{c m})$ & $\begin{array}{c}\text { Chloride } \\
(\mathrm{mg} / \mathrm{L})\end{array}$ & $\begin{array}{c}\text { TH } \\
(\mathrm{mg} / \mathrm{L})\end{array}$ & $\begin{array}{c}\text { Fluoride } \\
(\mathrm{mg} / \mathrm{L})\end{array}$ & $\begin{array}{c}\text { Iron } \\
(\mathrm{mg} / \mathrm{L})\end{array}$ & $\begin{array}{c}\text { Nitrate } \\
(\mathrm{mg} / \mathrm{L})\end{array}$ & $\begin{array}{c}\mathrm{RC} \\
(\mathrm{mg} / \mathrm{L})\end{array}$ & $\begin{array}{c}\text { Turbidity } \\
\text { (NTU) }\end{array}$ \\
\hline 1. & SW 1 & 8.67 & 283 & 566 & 70 & 150 & 1.0 & 0.3 & 10 & 0 & 5 \\
\hline 2. & SW 2 & 8.70 & 304 & 608 & 60 & 175 & 0.5 & 0.3 & 10 & 0 & 0 \\
\hline 3. & SW 3 & 7.80 & 204 & 408 & 50 & 100 & 1.0 & 0.0 & 45 & 0 & 0 \\
\hline 4. & SW 4 & 8.00 & 299 & 599 & 60 & 126 & 1.0 & 0.3 & 45 & 0 & 5 \\
\hline 5. & SW 5 & 8.20 & 270 & 541 & 50 & 124 & 0.5 & 0.3 & 45 & 0 & 0 \\
\hline 6. & SW 6 & 7.20 & 590 & 1181 & 120 & 200 & 1.5 & 0.3 & 10 & 0 & 5 \\
\hline 7. & SW 7 & 7.80 & 617 & 1235 & 130 & 208 & 1.0 & 0.3 & 10 & 0 & 5 \\
\hline 8. & SW 8 & 7.40 & 593 & 1186 & 70 & 276 & 1.0 & 0.3 & 10 & 0 & 5 \\
\hline 9. & SW 9 & 7.10 & 190 & 380 & 40 & 75 & 1.0 & 0.0 & 10 & 0 & 0 \\
\hline 10. & SW 10 & 7.20 & 203 & 406 & 40 & 106 & 0.5 & 0.0 & 10 & 0 & 5 \\
\hline 11. & SW 11 & 7.80 & 350 & 700 & 160 & 200 & 0.5 & 0.0 & 10 & 0 & 5 \\
\hline 12. & SW 12 & 8.90 & 980 & 1960 & 200 & 620 & 1.0 & 0.5 & 45 & 0 & 20 \\
\hline 13. & Mean & 7.897 & 406.916 & 814.166 & 87.5 & 196.667 & 0.875 & 0.216 & 21.666 & 0 & 4.583 \\
\hline
\end{tabular}

$\mathrm{SW}=$ surface water, $\mathrm{TDS}=$ total dissolved solid, $\mathrm{EC}=$ electrical conductivity, $\mathrm{TH}=$ total hardness, $\mathrm{RC}=$ residual chlorine

Table.4 Physico-chemical analysis of surface water in peri-urban areas of Udaipur

\begin{tabular}{|c|c|c|c|c|c|c|c|c|c|c|c|}
\hline $\begin{array}{l}\text { S. } \\
\text { No. }\end{array}$ & Sample No. & $\begin{array}{c}\mathbf{p H} \\
(\mathrm{mg} / \mathrm{L})\end{array}$ & $\begin{array}{c}\text { TDS } \\
(\mathrm{mg} / \mathrm{L})\end{array}$ & $\mathrm{EC}(\boldsymbol{\mu} \mathrm{s} / \mathrm{cm})$ & $\begin{array}{c}\text { Chloride } \\
\text { (mg/L) }\end{array}$ & $\begin{array}{c}\text { TH } \\
(\mathrm{mg} / \mathrm{L})\end{array}$ & $\begin{array}{c}\text { Fluoride } \\
\text { (mg/L) }\end{array}$ & $\begin{array}{c}\text { Iron } \\
(\mathrm{mg} / \mathrm{L})\end{array}$ & $\begin{array}{l}\text { Nitrate } \\
(\mathrm{mg} / \mathrm{L})\end{array}$ & $\begin{array}{c}\mathrm{RC} \\
(\mathrm{mg} / \mathrm{L})\end{array}$ & $\begin{array}{c}\text { Turbidity } \\
\text { (NTU) }\end{array}$ \\
\hline 1. & SW 13 & 8.38 & 957 & 1914 & 220 & 375 & 1.5 & 0.3 & 100 & 0 & 25 \\
\hline 2. & SW 14 & 8.62 & 1167 & 2334 & 210 & 375 & 1.0 & 0.5 & 10 & 0 & 25 \\
\hline 3. & SW 15 & 7.86 & 710 & 1420 & 190 & 325 & 0.5 & 0.3 & 45 & 0 & 0 \\
\hline 4. & SW 16 & 8.58 & 437 & 844 & 80 & 175 & 1.0 & 0.3 & 10 & 0 & 0 \\
\hline 5. & SW 17 & 7.18 & 431 & 862 & 70 & 206 & 0.5 & 0.3 & 10 & 0 & 0 \\
\hline 6. & SW 18 & 7.10 & 298 & 596 & 60 & 126 & 0.5 & 1.0 & 10 & 0 & 5 \\
\hline 7. & SW 19 & 7.71 & 640 & 1280 & 240 & 500 & 1.5 & 0.5 & 45 & 0 & 5 \\
\hline 8. & SW 20 & 7.20 & 370 & 740 & 110 & 325 & 0.5 & 0.3 & 10 & 0 & 5 \\
\hline 9. & SW 21 & 8.15 & 270 & 540 & 160 & 300 & 1.5 & 0.3 & 45 & 0 & 0 \\
\hline 10. & SW 22 & 7.80 & 250 & 500 & 130 & 250 & 1.5 & 1.0 & 10 & 0 & 0 \\
\hline 11. & Mean & 7.858 & 533 & 1103 & 147 & 295.7 & 1 & 0.48 & 29.5 & 0 & 6.5 \\
\hline
\end{tabular}


Table.5 Physico-chemical analysis of ground water in urban areas of Udaipur

\begin{tabular}{|c|c|c|c|c|c|c|c|c|c|c|c|}
\hline $\begin{array}{l}\text { S. } \\
\text { No. }\end{array}$ & Sample No. & $\begin{array}{c}\mathbf{p H} \\
(\mathbf{m g} / \mathrm{L})\end{array}$ & $\begin{array}{c}\text { TDS } \\
(\mathrm{mg} / \mathrm{L})\end{array}$ & $\mathrm{EC}(\mu \mathrm{s} / \mathrm{cm})$ & $\begin{array}{c}\text { Chloride } \\
\text { (mg/L) }\end{array}$ & $\begin{array}{c}\text { TH } \\
(\mathrm{mg} / \mathrm{L})\end{array}$ & $\begin{array}{c}\text { Fluoride } \\
(\mathrm{mg} / \mathrm{L})\end{array}$ & $\begin{array}{c}\text { Iron } \\
(\mathrm{mg} / \mathrm{L})\end{array}$ & $\begin{array}{l}\text { Nitrate } \\
(\mathrm{mg} / \mathrm{L})\end{array}$ & $\begin{array}{c}\mathrm{RC} \\
(\mathrm{mg} / \mathrm{L})\end{array}$ & $\begin{array}{c}\text { Turbidity } \\
\text { (NTU) }\end{array}$ \\
\hline 1. & GW 1 & 7.10 & 827 & 1665 & 180 & 375 & 1.5 & 0 & 10 & 0 & 0 \\
\hline 2. & GW 2 & 7.20 & 1310 & 2620 & 450 & 600 & 1.0 & 0 & 45 & 0 & 15 \\
\hline 3. & GW 3 & 7.30 & 1217 & 2434 & 330 & 275 & 2.0 & 0 & 10 & 0 & 0 \\
\hline 4. & GW 4 & 7.10 & 1622 & 3244 & 750 & 750 & 2.5 & 0.5 & 10 & 0 & 0 \\
\hline 5. & GW 5 & 7.42 & 721 & 1443 & 130 & 350 & 1.0 & 0.3 & 10 & 0 & 0 \\
\hline 6. & GW 6 & 8.10 & 335 & 670 & 70 & 150 & 0.5 & 0.5 & 10 & 0 & 0 \\
\hline 7. & GW 7 & 7.90 & 301 & 602 & 70 & 150 & 0.5 & 0 & 10 & 0 & 0 \\
\hline 8. & GW 8 & 7.80 & 1477 & 2954 & 300 & 625 & 1.5 & 0.3 & 45 & 0 & 0 \\
\hline 9. & GW 9 & 7.00 & 1130 & 2261 & 300 & 534 & 1.5 & 0.3 & 0 & 0 & 0 \\
\hline 10. & GW 10 & 7.10 & 1422 & 2844 & 330 & 424 & 1.5 & 0.3 & 10 & 0 & 0 \\
\hline 13. & Mean & 7.402 & 1036.2 & 2073.7 & 291 & 423.3 & 1.35 & 0.22 & 16 & 0 & 1.5 \\
\hline
\end{tabular}

$\mathrm{GW}=$ ground water, $\mathrm{TDS}=$ total dissolved solid, $\mathrm{EC}=$ electrical conductivity, $\mathrm{TH}=$ total hardness, $\mathrm{RC}=$ residual chlorine

Table.6 Physico-chemical analysis of ground water in peri-urban areas of Udaipur

\begin{tabular}{|c|c|c|c|c|c|c|c|c|c|c|c|}
\hline $\begin{array}{c}\text { S. } \\
\text { No. }\end{array}$ & Sample No. & $\begin{array}{c}\mathbf{p H} \\
(\mathbf{m g} / \mathbf{L})\end{array}$ & $\begin{array}{c}\text { TDS } \\
(\mathbf{m g} / \mathbf{L})\end{array}$ & $\begin{array}{c}\text { EC } \\
(\boldsymbol{\mu s} / \mathbf{c m})\end{array}$ & $\begin{array}{c}\text { Chloride } \\
(\mathbf{m g} / \mathbf{L})\end{array}$ & $\begin{array}{c}\text { TH } \\
(\mathbf{m g} / \mathbf{L})\end{array}$ & $\begin{array}{c}\text { Fluoride } \\
(\mathbf{m g} / \mathbf{L})\end{array}$ & $\begin{array}{c}\text { Iron } \\
(\mathbf{m g} / \mathbf{L})\end{array}$ & $\begin{array}{c}\text { Nitrate } \\
(\mathbf{m g} / \mathbf{L})\end{array}$ & $\begin{array}{c}\text { RC } \\
(\mathbf{m g} / \mathbf{L})\end{array}$ & $\begin{array}{c}\text { Turbidity } \\
(\mathbf{N T U})\end{array}$ \\
\hline $\mathbf{1 .}$ & GW 11 & 7.86 & 2180 & 4360 & 910 & 1025 & 1.0 & 0.3 & 10 & 0 \\
\hline 2. & GW 12 & 7.55 & 2715 & 5430 & 1100 & 1925 & 1.0 & 0.3 & 45 & 0 \\
\hline 3. & GW 13 & 6.92 & 1858 & 3317 & 850 & 1250 & 1.0 & 0.3 & 10 & 0 \\
\hline 4. & GW 14 & 6.80 & 1195 & 2390 & 450 & 625 & 1.0 & 0 & 10 & 0 \\
\hline $\mathbf{5 .}$ & GW 15 & 6.81 & 1643 & 3286 & 550 & 708 & 1.0 & 0.3 & 10 & 0 \\
\hline 6. & GW 16 & 7.00 & 1300 & 2600 & 350 & 530 & 1.5 & 0.3 & 10 & 0 \\
\hline 7. & GW 17 & 8.70 & 1032 & 2064 & 220 & 625 & 2.5 & 0.3 & 10 & 0 \\
\hline 8. & GW 18 & 6.90 & 1109 & 2218 & 250 & 725 & 1.5 & 0.3 & 10 & 0 \\
\hline 9. & GW 19 & 7.45 & 1620 & 3240 & 650 & 700 & 1.5 & 0.3 & 10 & 0 \\
\hline 10. & GW 20 & 7.28 & 1814 & 3629 & 800 & 625 & 1.0 & 0 & 10 & 0 \\
\hline 11. & Mean & 7.297 & 1646.6 & 3253.4 & 613 & 873.8 & 1.3 & 0.24 & 13.5 & 0 \\
\hline
\end{tabular}


Table.7 Physico-chemical analysis of animal drinking water in urban areas of Udaipur

\begin{tabular}{|c|c|c|c|c|c|c|c|c|c|c|c|}
\hline $\begin{array}{l}\text { S. } \\
\text { No. }\end{array}$ & Sample No. & $\begin{array}{c}\mathbf{p H} \\
(\mathbf{m g} / \mathrm{L})\end{array}$ & $\begin{array}{c}\text { TDS } \\
(\mathrm{mg} / \mathrm{L})\end{array}$ & $\begin{array}{c}\text { EC } \\
(\mu \mathrm{s} / \mathbf{c m})\end{array}$ & $\begin{array}{c}\text { Chloride } \\
\text { (mg/L) }\end{array}$ & $\begin{array}{c}\text { TH } \\
(\mathrm{mg} / \mathrm{L})\end{array}$ & $\begin{array}{c}\text { Fluoride } \\
(\mathrm{mg} / \mathrm{L})\end{array}$ & $\begin{array}{l}\text { Iron } \\
(\mathrm{mg} / \mathrm{L})\end{array}$ & $\begin{array}{c}\text { Nitrate } \\
(\mathrm{mg} / \mathrm{L})\end{array}$ & $\begin{array}{c}\text { RC } \\
(\mathrm{mg} / \mathrm{L})\end{array}$ & $\begin{array}{c}\text { Turbidity } \\
\text { (NTU) }\end{array}$ \\
\hline 1. & AW 1 & 7.20 & 827 & 1665 & 180 & 350 & 1.5 & 0 & 10 & 0 & 0 \\
\hline 2. & AW 2 & 6.82 & 1321 & 2642 & 550 & 675 & 2.5 & 0.3 & 45 & 0 & 0 \\
\hline 3. & AW 3 & 7.20 & 1212 & 2424 & 330 & 275 & 2.0 & 0 & 10 & 0 & 15 \\
\hline 4. & AW 4 & 6.91 & 1639 & 3279 & 800 & 800 & 2.5 & 0.5 & 10 & 0 & 25 \\
\hline 5. & AW 5 & 7.30 & 710 & 1420 & 140 & 350 & 1.0 & 0.3 & 10 & 0 & 5 \\
\hline 6. & AW 6 & 7.89 & 700 & 1401 & 130 & 375 & 1.0 & 0.3 & 10 & 0 & 0 \\
\hline 7. & AW 7 & 8.16 & 378 & 757 & 80 & 175 & 0.5 & 0 & 10 & 0 & 0 \\
\hline 8. & AW 8 & 7.80 & 1408 & 2816 & 290 & 575 & 1.0 & 0 & 45 & 0 & 5 \\
\hline 9. & AW 9 & 7.20 & 1335 & 2670 & 420 & 584 & 1.0 & 0 & 10 & 0 & 5 \\
\hline 10. & AW 10 & 6.80 & 1167 & 2234 & 420 & 425 & 1.5 & 0.3 & 45 & 0 & 5 \\
\hline 11. & Mean & 7.328 & 1069.7 & 2130.8 & 334 & 458.4 & 1.45 & 0.17 & 20.5 & 0 & 6 \\
\hline
\end{tabular}

$\mathrm{AW}=$ animal drinking water, $\mathrm{TDS}=$ total dissolved solid, $\mathrm{EC}=$ electrical conductivity, $\mathrm{TH}=$ total hardness, $\mathrm{RC}=$ residual chlorine

Table.8 Physico-chemical analysis of animal drinking water in peri-urban areas of Udaipur

\begin{tabular}{|c|c|c|c|c|c|c|c|c|c|c|c|}
\hline $\begin{array}{c}\text { S. } \\
\text { No. }\end{array}$ & Sample No. & $\begin{array}{c}\mathbf{p H} \\
(\mathbf{m g} / \mathbf{L})\end{array}$ & $\begin{array}{c}\text { TDS } \\
(\mathbf{m g} / \mathbf{L})\end{array}$ & $\begin{array}{c}\mathbf{E C} \\
(\boldsymbol{\mu} \mathbf{s} / \mathbf{c m})\end{array}$ & $\begin{array}{c}\text { Chloride } \\
(\mathbf{m g} / \mathbf{L})\end{array}$ & $\begin{array}{c}\mathbf{T H} \\
(\mathbf{m g} / \mathbf{L})\end{array}$ & $\begin{array}{c}\text { Fluoride } \\
(\mathbf{m g} / \mathbf{L})\end{array}$ & $\begin{array}{c}\text { Iron } \\
(\mathbf{m g} / \mathbf{L})\end{array}$ & $\begin{array}{c}\text { Nitrate } \\
(\mathbf{m g} / \mathbf{L})\end{array}$ & $\begin{array}{c}\text { RC } \\
(\mathbf{m g} / \mathbf{L})\end{array}$ & $\begin{array}{c}\text { Turbidity } \\
(\mathbf{N T U})\end{array}$ \\
\hline $\mathbf{1 .}$ & AW 11 & 7.91 & 2229 & 4459 & 950 & 1125 & 1.5 & 0.3 & 45 & 0 \\
\hline $\mathbf{2 .}$ & AW 12 & 7.92 & 833 & 1667 & 70 & 525 & 1.5 & 0.3 & 10 & 0 \\
\hline 3. & AW 13 & 7.76 & 1819 & 3639 & 850 & 1250 & 1.0 & 0.3 & 10 & 0 \\
\hline 4. & AW 14 & 6.90 & 1260 & 2520 & 450 & 700 & 1.0 & 0.3 & 10 & 0 \\
\hline 5. & AW 15 & 7.14 & 1711 & 3422 & 550 & 762 & 1.0 & 0.3 & 10 & 0 \\
\hline 6. & AW 16 & 6.90 & 1350 & 2700 & 350 & 560 & 1.0 & 0.3 & 10 & 0 \\
\hline 7. & AW 17 & 7.58 & 903 & 1806 & 130 & 500 & 2.5 & 0.3 & 10 & 0 \\
\hline 8. & AW 18 & 7.10 & 1132 & 2264 & 250 & 700 & 1.0 & 0.3 & 10 & 0 \\
\hline 9. & AW 19 & 7.65 & 1580 & 3160 & 600 & 675 & 1.0 & 0.3 & 10 & 0 \\
\hline 10. & AW 20 & 8.15 & 1340 & 2680 & 550 & 500 & 2.0 & 0.3 & 45 & 0 \\
\hline 11. & Mean & 7.501 & 1415.7 & 2831.7 & 475 & 729.7 & 1.35 & 0.3 & 17 & 0 \\
\hline
\end{tabular}


Fig.1 Variation of TDS, chloride content and total hardness of different types of water

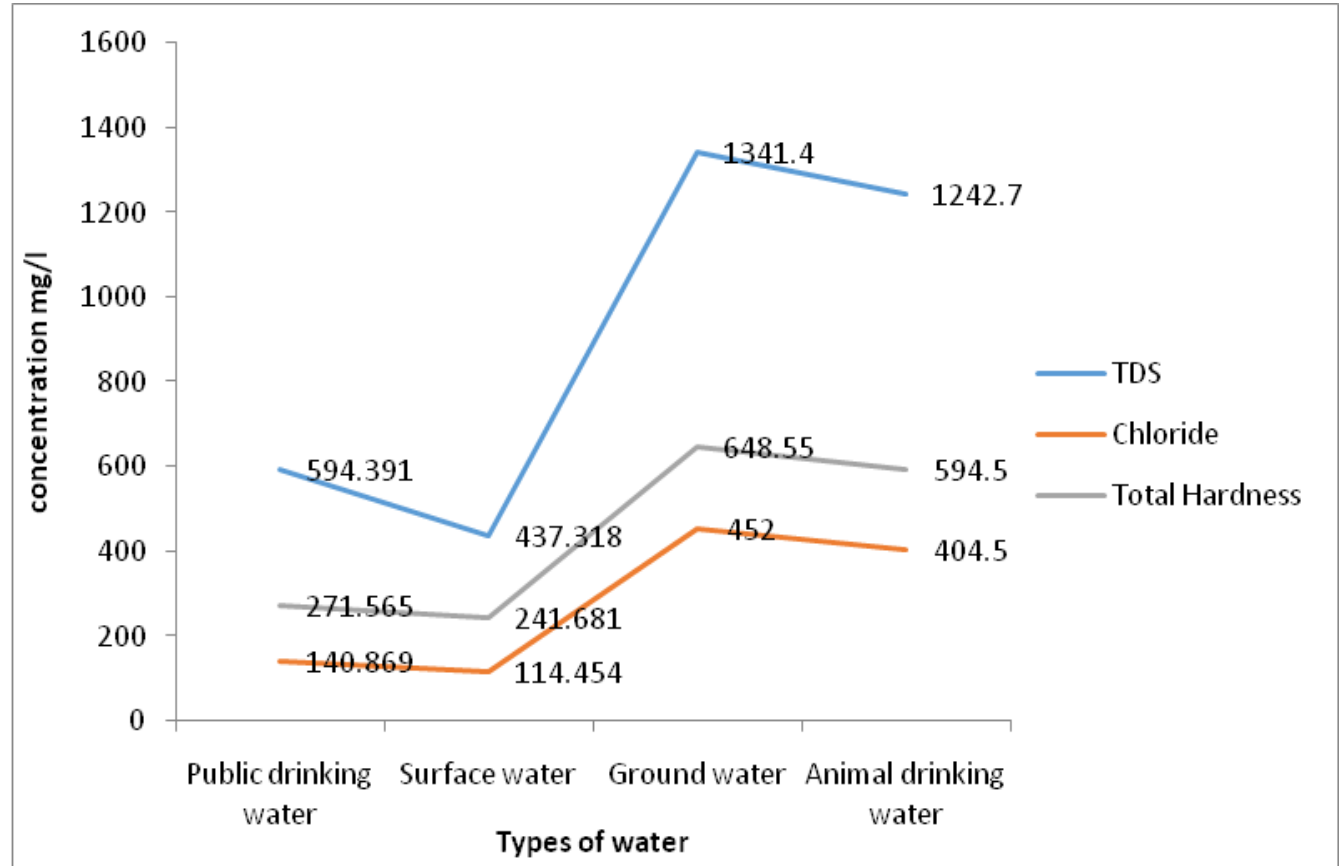

Fig.2 Variation of fluoride, iron and nitrate content in different types of water

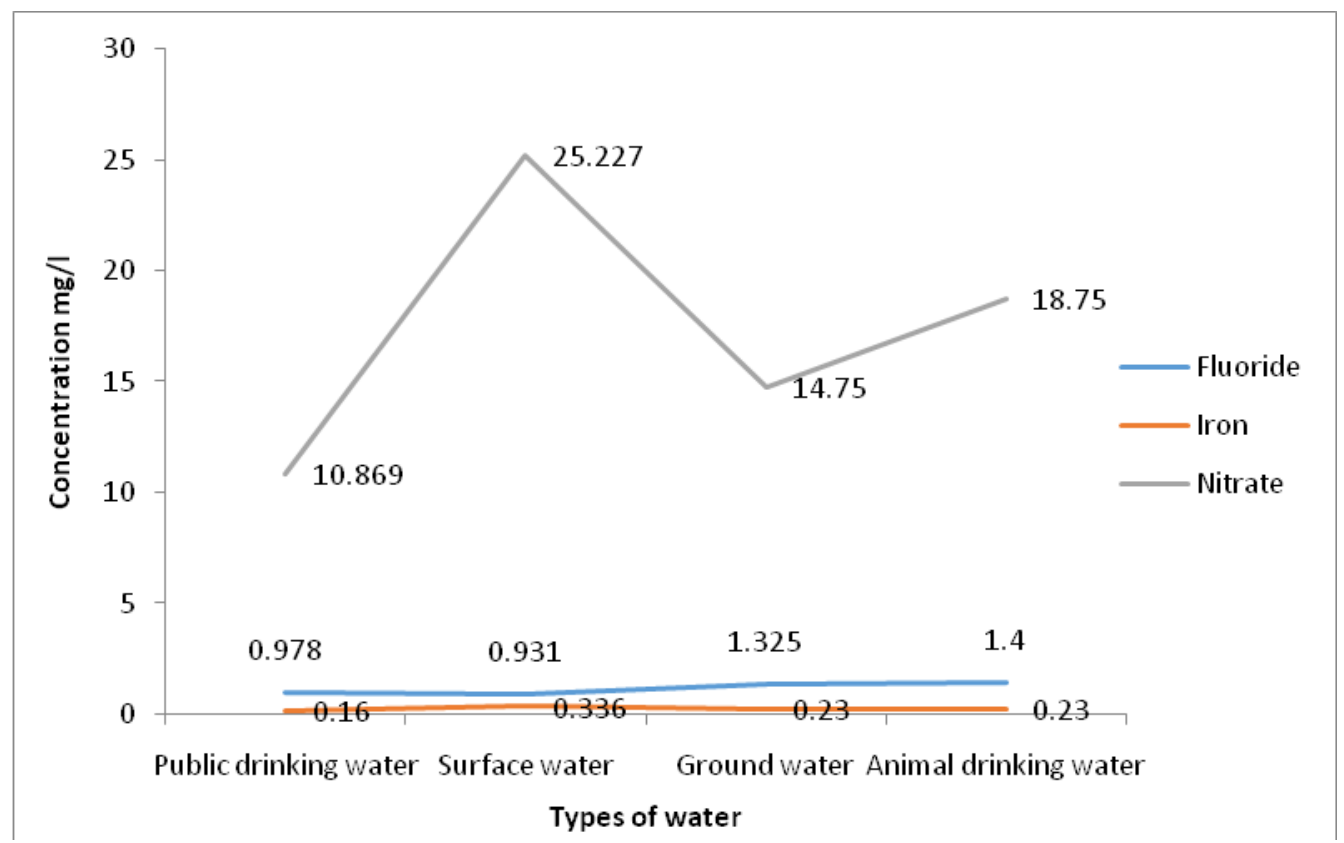

One of the most vital inorganic ions in water is chloride. This is found in almost all water bodies as it is highly soluble. It is also regarded as an indicator of sewage
pollution(Wetzel, 1966).Chlorides are leached from various rocks into soil and water by weathering (WHO, 1996). Chloride values found in the range between $10 \mathrm{mg} / \mathrm{l}$ to 1100 
$\mathrm{mg} / \mathrm{l}$ in different categories of water. In which urban areas ranged found in between $10 \mathrm{mg} / \mathrm{l}$ to $800 \mathrm{mg} / \mathrm{l}$ while, in peri-urban areas ranged found between $50 \mathrm{mg} / \mathrm{l}$ to $1100 \mathrm{mg} / \mathrm{l}$ (Table 1 to 8; Fig. 1 and 2). Slightly lower chloride content in drinking water were reported by Sebiawu et al., 2014 and Chaubey and Patil 2015.All category of water chloride comes in acceptable limit for drinking water is $250 \mathrm{mg} / \mathrm{l}$ to1100mg/l (BIS, 2012).

Fluoride values found in the range between 0 $\mathrm{mg} / \mathrm{l}$ to $2.5 \mathrm{mg} / \mathrm{l}$ in different categories of water. In which urban areas ranged found in between $0 \mathrm{mg} / \mathrm{l}$ to $2.5 \mathrm{mg} / \mathrm{l}$ while, in periurban areas ranged found between $0.5 \mathrm{mg} / \mathrm{l}$ to $2.5 \mathrm{mg} / \mathrm{l}($ Table 1 to 8; Fig. 1 and 2). Many ground water samples show high contain of fluoride due to the nature of specific structure of the rocks and soil of particular area. Similar to our finding for fluoride content in drinking water were reported by Reda, 2016. Fluoride recommendation for drinking water is $1 \mathrm{mg} / \mathrm{l}$ to $1.5 \mathrm{mg} / \mathrm{l}$. (BIS, 2012).

Iron values found in the range between $0 \mathrm{mg} / \mathrm{l}$ to $1.0 \mathrm{mg} / \mathrm{l}$ in different categories of water. In which urban areas ranged found in between 0 $\mathrm{mg} / \mathrm{l}$ to $0.5 \mathrm{mg} / \mathrm{l}$ while, in peri-urban areas ranged found between $0 \mathrm{mg} / \mathrm{l}$ to 1.0 $\mathrm{mg} / \mathrm{l}$ (Table 1 to 8 ; Fig. 1 and 2).Sebiawu et al., 2014 and Rahmania et al., 2015 revealed iron content of drinking water which were in agreement with the findings of our study.Iron recommendation for drinking water is 0.3 $\mathrm{mg} / \mathrm{l}$. (BIS, 2012).

Nitrate values found in the range between 0 $\mathrm{mg} / \mathrm{l}$ to $100 \mathrm{mg} / \mathrm{l}$ in different categories of water. In which urban areas ranged found in between $0 \mathrm{mg} / \mathrm{l}$ to $45 \mathrm{mg} / \mathrm{l}$ while, in periurban areas ranged found between $0 \mathrm{mg} / \mathrm{l}$ to $100 \mathrm{mg} / \mathrm{l}$ (Table 1 to 8; Fig. 1 and 2).Singh et al., 2014 and Adegboyega et al., 2015 also found similar to our results for nitrate content in water. Nitrate is produced by the action of microbes on fertilizers. The increased use of fertilizer in agriculture practice may be an important source of nitrate contamination in water. Leaching of fertilizers to the water table through the soil is also an important means of ground water contamination. Nitrate recommendation for drinking water is 45 $\mathrm{mg} / \mathrm{l}$. (BIS, 2012)

All these parameters chloride, fluoride, iron and nitrate were in the acceptable range recommended by BIS. High levels of iron occur due to specific structure of the rocks in the study area. Moreover, the salty taste in water occurs due to the high chloride concentration. Increased amount of chloride, fluoride and iron indicates the role of anthropogenic activities and sewage pollution as the cause of their contamination in drinking water.

Turbidity of water is an important parameter which is directly linked with the increased amount of organic matter in the water. It is not only hazardous for human health but also adversely affects the efficacy of disinfectant. Also, turbid water has high concentration of pathogenic microbes like bacteria and other parasites which pose a serious health hazard. In our study, few samples of surface water exceeded the acceptable limit of turbidity. Turbidity values found in the range between 0 NTU to 25 NTU in different categories of water. In which urban areas ranged found in between 0 NTU to 25 NTU while, in periurban areas ranged found between 0 NTU to 25 NTU. Turbidity recommendation for drinking water is not more than 1.0 NTU. (BIS, 2012)And all of the water samples were negative for residual chlorine(Table 1 to 8 ; Fig. 1 and 2).

\section{Acknowledgement}

I am thankful to co-others Dr. Abhishek Guarav, Dr. S.S. Shekhawat, Dr. Bincy 
Joseph, Dr. Hitesh Kumar and Dr. Devender Choudhary, Department of Veterinary public Health, CVAS, Navania, Vallabhnagar, Udaipur for their gaudiness, co-operation and persistence motivation during entire period of study.

\section{References}

Acharya G.D., Hathi M.V., Patel A.D.andParmar K.C. (2008). Chemical properties of groundwater in BhilodaTaluka Region, North Gujarat. India. E-Journal of Chemistry 5(4): 792-796.

Adegboyega A.M., Olalude C.B. and Odunola O.A. (2015). Physicochemical and Bacteriological Analysis of Water Samples Used For Domestic Purposes in Idi Ayunre, Oyo State, Southwestern Nigeria. Journal of Applied Chemistry8(10): 46-50.

Ademola F. (2008). Baseline heavy metals concentration in river sediments with Okikipopo South East belt of the Nigerian bituminious sand field. $J$ ChemSoc Nig. 33.

American Public Health Association. (2005). Standard method for examination of water and wastewater, NW, DC 20036.

Australian and New Zealand Environment and Conservation Council. (2000). Water Quality Guidelines, 2000.

Bureau of Indian Standards. (2012). IS 10500: Indian standard drinking waterspecification, Second revision: 1-11.

Buridi K.R. and Gedala R.K. (2014). Study on Determination of Physicochemical Parameters of Ground Water in Industrial Area of Pydibheemavaram, Vizianagaram District, Andhra Pradesh, India. Austin Journal of Public Health and Epidemiology 1(2): 1-2.

Chaubey S. and Patil M.K. (2015). Correlation Study and Regression Analysis of Water Quality Assessment of Nagpur City, India.International Journal of Scientific and Research Publications11(5): 753-757.

Chidinma I., Matthew O., Grace E., Emmanuel N, Chika E., Ifeanyichukwu I., Monique M. and Emeka I. (2016). Bacteriological and physicochemical parameters of some selected borehole water sources in Abakaliki metropolis, Nigeria.Int J Community Med Public Health3(11): 3271-3277.

Chindo I.Y., Karu E., Ziyok I. and Amanki E.D. (2013). Physicochemical Analysis of Ground Water of Selected Areas of Dass and Ganjuwa Local Government Areas, Bauchi State, Nigeria. World Journal of Analytical Chemistry 1(4): 73-79.

Dixit A.K., Pandey S.K., Mehta R., Ahmad N., Gunjan and Pandey J. (2015). Study of physico-chemical parameters of different pond water of Bilaspur District, Chhattishgarh, India. Environmental Skeptics and Critics. 4(3): 89-95.

Ehiowemwenguan G., Iloma A.O. and Adetuwo J.O. (2014). PhysicoChemical and Bacteriological Quality of Borehole Water in Eyaen Community Area of Edo State, Nigeria. International Journal of Basic and Applied Science3(2): 60-68.

Ghosh B.B. (2018). Physicochemical analysis of pond water in purbabarddhaman, West Bengal, India. Int. Res. J. Environmental Sci.7(2): 54-59.

Hassan M., Islam S.M.D., Ahmed F. and Rahman M.A.T.M.T. (2016). Quality Analysis of Drinking Water Provided for the Readymade Garment Workers in Dhaka, Bangladesh. Pollution2(3): 289298.

Mostafa A.M., Al-Wasify R.S., Sayed A.M. and Haroun B.M. (2013) Microbiological and Physicochemical Evaluation of Groundwater in 
Egypt.International Journal of Environment and Sustainability 2(2): 110.

Olatayo A.A. (2014). Assessment of PhysicoChemical Parameters of Waters in Ilaje Local Government Area of Ondo State, Nigeria. International Journal of Fisheries and Aquatic Studies 1(5): 8492.

Parvez A.K., Liza S.M. and Marzan M. (2016). Bacteriological Quality of Drinking Water Samples across Bangladesh. Arch. Clin. Microbiol. 7: $1-9$

Rahmanian N., Ali S.H.B., Homayoonfard M., Ali N.J., Rehan M., Sadef Y. and Nizami A.S. (2015). Analysis of Physiochemical Parameters to Evaluate the Drinking Water Quality in the State of Perak, Malaysia. Journal of Chemistry. 1-10.

Rai N.K. and Chouhan P. (2017). PhysicoChemical Analysis for Different Lakes/River of Udaipur City- A Case Study. International Journal of Scientific \& Engineering Research10(8): 612-617.

Ramachandraiah C. (2004). Right to drinking water in India, Centre for Economic and Social Studies 56.

Rani J., Anita Kannagi, Shanthi V, Correlation of total heterotrophic bacterial load in relation with hydrographical features of Pazhayakayal estuary, Tuticorin, India., J. Environ. Biol., 2012; 33:769-773.

Reda A.H. (2016). Physico-Chemical Analysis of Drinking Water Quality of Arbaminch Town. $J$ Environ Anal Toxicol.6(2): 356.

Saha R.,Dey N.C.,Rahman S.,Galagedara L. and Bhattacharya P. (2018). Exploring suitable sites for installing safe drinking water wells in coastal Bangladesh. Groundwater for Sustainable Development.7: 91-100.
Samuel O., Rosemary E., Patrice O. and Frederick O. (2017). An Evaluation of the Physicochemical Characteristics of the Hand-Dug Shallow Water Wells in Awka Metropolis, Anambra State, Nigeria. American Journal of Life Science Researches5(3): 89-101.

Sangu R.P.S. and Sharma S.K. (1987). An assessment of water quality of river Ganga at Garmukeshwar. Ind. J. Ecol. 14(20): 278-287.

Sebiawu G E., Fosu S.A. and Saana S.B.B.M. (2014). Dispensing Technology Department, Wa Polytechnic, Wa. A Physico-Chemical and Bacteriological Analysisof Borehole Water Samples from the WaMunicipality of the Upper West Region, Ghana. International Journal of Engineering Research \& Technology3(5).

Sebiawu G E., Fosu S.A. and Saana S.B.B.M. (2014). Dispensing Technology Department, Wa Polytechnic, Wa. A Physico-Chemical and Bacteriological Analysisof Borehole Water Samples from the WaMunicipality of the Upper West Region, Ghana. International Journal of Engineering Research \& Technology3(5).

Shukla D., Bhadresha K., Jain N.K. and Modi H.A. (2013). Physicochemical Analysis of Water from Various Sources and Their Comparative Studies IOSR Journal of Environmental Science, Toxicology and Food Technology 5(3): 89-92.

Singh S., Negi R.S. and Dhanai R. (2014). A study of physico-chemical parameters of springs around Srinagar Garhwal valley, Uttarakhand. International Journal of Engineering Development and Research2(4): 3885-3887.

Sunday J.J., Spencer N.C.O., Kingsley O., Edet A.O. and Amaka D.D. (2014).Physico-chemical and microbiological properties of water 
samples used for domestic purposes in Okada town, Edo state, Nigeria. Int. J. Curr. Microbiol. App. Sci. 3(6): 886894.

Sunitha D., Murthy S.M., Divya K.S. and Ramalingam A. (2013). Assessment of Physico-chemical and Bacteriological Parameters of Drinking Water from Different Sources in Mysore City. International Journal of Innovative Research in Science, Engineering and Technology.

Ubalua O.A and Ezeronye O.U. (2005). Nutrients and selected Physicochemical analysis in the ABA rivers surface waters Abia State, Nigeria. Environment and Ecology 23 (1): 141144.

Vyas V.G., Hassan M.H., Vindhani S.I., Parmar H.J. and Bhalani V.M. (2015).Physicochemical and Microbiological Assessment of Drinking Water from Different Sources in Junagadh City, India. American
Journal of Microbiological Research3(4): 148-154.

Wetzel R.G. (1966). Variations in productivity of Goose and hypereutrophicSylvian Lakes, Indiana. Indiana Lakesand Strems 7: 147-184.

WHO (World Health Organization) (1997). Guideline for drinking water quality, 2nd ed., Vol 2 Health criteria and other supporting information, World Health organization, Geneva 940-949.

WHO. (1996). Health criteria and other supporting information; second ed. Guidelines for drinking water quality, Vol. 2. World Health Organization. Geneva. Switzerland.

World Health Organization.(2017).Guidelines for drinking-water quality, 4th edition, incorporating the $1^{\text {st }}$ addendum.

World Health Organization. (2010). Guidelines for Drinking water Quality. Recommendation, Geneva. 1-6.

\section{How to cite this article:}

Nirmal Kumar, Abhishek Gaurav, Surendra Singh Shekhawat, Bincy Joseph, Hitesh Kumar and Devender Choudhary. 2019. Physico-Chemical Assessment of Drinking Water in Urban and Peri-Urban Areas of Udaipur, India. Int.J.Curr.Microbiol.App.Sci. 8(08): 2314-2326. doi: https://doi.org/10.20546/ijcmas.2019.808.268 